\title{
Strategies for Management of Synchronous Colorectal Metastases
}

\author{
Jason A. Castellanos $\cdot$ Nipun B. Merchant
}

Published online: 20 June 2014

(C) Springer Science + Business Media New York 2014

\begin{abstract}
The management of synchronous presentation of colorectal cancer and liver metastases has long been a topic of debate and discussion for surgeons due to the unique dilemma of balancing operative timing along with treatment strategy. Operative strategies for resection include staged resection with colon first approach, "reverse" staged resection with liver metastases resected first, and one-stage, or simultaneous, resection of both the primary tumor and liver metastases approach. These operative strategies can be further augmented with perioperative chemotherapy and other novel approaches that may improve resectability and patient survival. The decision on operative timing and approach, however, remains largely dependent on the surgeon's determination of disease resectability, patient fitness, and the need for neoadjuvant chemotherapy.
\end{abstract}

This article is part of the Topical Collection on Colorectal Liver Metastases.

\section{J. A. Castellanos · N. B. Merchant}

Division of Surgical Oncology and Endocrine Surgery,

Vanderbilt University Medical Center, Nashville, TN, USA

e-mail: jason.castellanos@vanderbilt.edu

\section{J. A. Castellanos}

Department of Surgery, Vanderbilt University Medical Center,

1161 21st Ave S., D2300 MCN, Nashville, TN 37232, USA

\section{N. B. Merchant $(\square)$}

Department of Surgery and Cancer Biology, Vanderbilt

University Medical Center, 597 Preston Research Building,

2220 Pierce Ave, Nashville, TN 37232, USA

e-mail: nipun.merchant@vanderbilt.edu
Keywords Colorectal cancer $\cdot$ CRC $\cdot$ Metastases · Synchronous liver metastasis - sCRLM .

Lymphadenopathy · Hepatectomy · Chemotherapy · Chemoradiation

\section{Introduction}

Colorectal cancer (CRC) will account for over 136,000 new cases and 50,310 deaths in 2014 [1]. The liver is the most common site of metastasis, and up to $25 \%$ of newly diagnosed patients may present with synchronous liver metastasis (sCRLM) [2]. The management of sCRLM has long been a topic of debate for surgeons due to the unique dilemma of balancing operative timing and strategy. This has been made manifestly more complicated with the emergence of efficacious systemic chemotherapy regimens, targeted biological agents, and adjunctive strategies (e.g., ablative therapies). Additionally, the surgical approaches to disease extirpation have become more aggressive as advances in critical care and anesthesia have decreased operative morbidity and mortality substantially. Confronted with this clinical scenario, the surgeon must answer three central questions:

(1) Should the primary and metastatic lesions be resected concurrently?

(2) If surgical resection is staged, should the primary or metastatic lesion be resected first?

(3) What is the role of perioperative chemotherapy with surgical resection?

In this review, we will present the current evidence for each of these three main strategies for resection ("conventional" staged resection with colon resected first approach, "reverse" staged resection with liver metastases 
resected first, and one-stage, or simultaneous resection of both the primary tumor and liver metastases) in addition to discussion of the role of perioperative chemotherapy and other novel strategies that may be required to improve resectability and patient survival.

\section{Determination of Resectability}

As is true of much in this field, the criteria defining resectable disease have significantly changed in recent years. Older criteria dictated that patients have fewer than four unilobar metastases, no extrahepatic disease and have resection margins greater than one centimeter. These criteria have given way to a paradigm where the primary consideration for resectability is the ability to gain a complete (R0) resection while maintaining adequate liver function. Modern criteria for determination of the resectability of liver metastatic disease were outlined by the Consensus Conference on Multidisciplinary Treatment of Colorectal Cancer Liver Metastases in 2012 [3••]. However, these guidelines continue to evolve and may differ between centers.

During the course of pre-operative staging, radiologic evaluation should provide the number and segmental distribution of sCRLMs in order to determine surgical resectability. With the improved accuracy of combined computed tomography (CT)-positron emission

Table 1 Guidelines for hepatic resection of colorectal liver metastases as outlined by the consensus conference on multidisciplinary treatment of colorectal cancer liver metastases on January 18, 2012

Oncologic criteria of resectability

Pretreatment radiologic staging evaluation

If there is extrahepatic disease, it is:

(1) Limited and amenable to surgical resection

(2) Or there is reasonable expectation of long-term control with adjuvant treatment

Defer resection if significant progression ${ }^{\mathrm{a}}$ of metastatic disease during treatment until disease is controlled with second

Technical criteria of resectability

Ability to remove all macroscopic tumor (i.e., R0 resection)

Ability to spare two contiguous liver segments

Ability to preserve adequate vascular inflow, outflow and biliary drainage

Ability to preserve an adequate $\mathrm{FLR}^{\mathrm{b}}$

If projected FLR volume is marginal and/or patient has underlying liver disease, carefully assess FLR based on regenerative response after portal vein embolization (PVE)

\footnotetext{
${ }^{a}$ Significant progression here refers to enlargement of more than three existing colorectal liver metastases and/or the development of multiple new lesions during optimal neo-adjuvant chemotherapy

b $20 \%$ of a normal liver, $30 \%$ of a liver in patients treated with chemotherapy
}

tomography, liver contrast-enhanced magnetic resonance imaging, and high resolution CT, the ability to detect small volume disease has been significantly enhanced [14]. The oncologic and technical criteria used to determine resectability are outlined in Table 1 . Involvement of celiac and/or para-aortic lymph nodes is considered a poor prognostic marker, and patients with this finding will likely not benefit from hepatectomy [4•]. While patients with portal and/or retropancreatic lymphadenopathy also have a worse prognosis, their survival outcomes are significantly better than patients with celiac lymphadenopathy, indicating a potential role for resection in this population of patients particularly with the use of perioperative chemotherapy [5•]. These criteria highlight the need for a multidisciplinary team to help guide each patient through the range of therapeutic options and determine the timing of resection of the primary and sCRLM. Strong consideration should be given to referral of these patients to a specialized center given the complexity of treatment planning.

\section{Operative Timing and Approach}

Without resection, median overall survival (OS) for patients with CRLM ranges from 20 to 24 months with modern chemotherapeutic regimens. With an R0 resection of all metastatic disease, 5-year OS has been reported to be as high as $58 \%[6,7 \bullet \cdot]$. Liver resection, therefore, remains the most efficacious treatment strategy for achieving longterm OS and the only potentially curative option for CRLM $[8 \cdot, 9 \bullet]$.

While there is an abundance of evidence demonstrating the benefit of hepatectomy for CRLM, there is only limited evidence available to empirically guide timing of resection for sCRLMs, and therefore this clinical scenario is primarily managed on a case-by-case basis. The conventional paradigm has been to first resect the primary tumor, and then perform a hepatectomy in a delayed fashion. This approach has the advantage of potentially avoiding complications associated with a larger, combined operation and also allows time for aggressive biology to manifest during the period in between operations to potentially save the patient from the extra morbidity of the hepatectomy [10, 11.]. This rationale was bolstered by observations that delaying resection did not appear to increase the risk of unresectability due to interval growth of CRLM, but rather due to the interval appearance of new liver and/or distant metastases [12•]. Additionally, the delay between operations allows for treatment with systemic chemotherapy that may decrease the risk of the appearance of new metastatic lesions and potentially improve OS [13•]. However, delayed hepatectomy may lead to increased hospitalization 
and additional cost, as well as increased pain, anxiety and stress for patients as they endure two instead of one major surgical procedure $\left[15,16 \bullet^{\circ}\right]$.

Given the declining morbidity associated with hepatectomy, simultaneous resection of both disease sites remains a viable option in appropriately selected patients. There is a growing body of literature demonstrating similar shortterm and long-term outcomes in patients undergoing simultaneous resection compared with patients undergoing delayed resection, even when a major hepatectomy (e.g., lobectomy) is performed $\left[17 \bullet, 18,19 \bullet, 20^{\bullet}\right]$ These studies contradict findings from other studies suggesting that operative mortality was significantly higher for those treated with simultaneous operations, particularly with major hepatectomies $[11 \bullet, 21 \bullet, 22]$. A recent multi-institutional retrospective analysis of 1,004 patients treated for sCRLM between 1982 and 2011 found no difference in postoperative complications or 90 day postoperative mortality between staged or simultaneous resection [23••]. In this study, a major hepatectomy was defined as resection of more than three segments, and the authors did observe that this was more commonly performed with a staged approach (39\%) versus a simultaneous approach $(24 \%)$.

These conflicting results highlight the gaps in knowledge currently present in this field. The lack of prospectively performed studies has necessitated the reliance on retrospective analyses of single or multi-institutional experiences and the potential interaction of selection bias with these results is strong. A recent meta-analyses by Slesser et al. [24••] compared the outcome of simultaneous and staged resections using 3,159 patients from studies published between 1991 and 2010. They found that while there were no significant differences in operative blood loss, duration of operation, post-operative complications, OS, or disease free survival (DFS), the 1,778 (56.3\%) of patients that underwent delayed resection had significantly larger liver metastases, with increased bilobar distribution and more of these patients underwent a major liver resection. Yin et al. [25*0] performed a meta-analysis of studies including a total of 2,880 patients, and their findings were similar, although they did note a significantly lower incidence of post-operative complications in the simultaneously resected group. Of note, based on this analysis Yin et al. [25••] recommended the following criteria for selection of patients to undergo simultaneous resection: liver resection involving less than four segments, age less than 70 and exclusion of patients with severe comorbid conditions. Both of these meta-analyses confirm that in the absence of clear clinical criteria to guide surgical decision making toward delayed or simultaneous resection, the surgeon's judgment, tempered by the counsel of a multidisciplinary committee and the patient's overall fitness for major abdominal surgery, remains the primary determinant of treatment strategy. Table 2 provides an overview of study characteristics for many of the studies included in these analyses in addition to more recent studies.

An alternative to these two approaches is the reverse, or liver first, staged resection in which the hepatectomy is performed prior to excision of the primary tumor. This approach is most often utilized for patients with rectal primary tumors as the timing dovetails conveniently with the waiting period after neoadjuvant chemoradiation therapy for the primary tumor has been completed. An added benefit is the avoidance of operative delay due to potential septic complications associated with resection of the primary rectal tumor. The liver first-staged approach can also be preceded by neoadjuvant chemotherapy specific for the metastatic lesions $[15,26 \bullet, 27]$. This strategic therapeutic delay offers the theoretical advantage of allowing the tumor biology to manifest, which in turn may indicate the suitability of aggressive resection. In addition to the risk of interval progression during initial neoadjuvant chemotherapy, there is also the potential for chemotherapy-associated parenchymal damage that may complicate efforts to ensure an adequate future liver remnant (FLR) and necessitate the use of adjunctive techniques, such as portal vein embolization or ligation to enhance FLR.

If there is a robust response to treatment and metastases are no longer visualized on radiographic studies, these lesions still need to be excised as the rate of complete pathologic response is low and the risk of regrowth remains high [26•].

The outcomes of the liver first approach have been detailed in small case series as well as larger multi-institutional reviews that indicate that liver first staged resection is feasible with similar outcomes to either simultaneous or colon first staged resection [15, 23••, 28, 29].

\section{The Role of Perioperative Systemic Chemotherapy}

Despite the improvements in surgical approaches of liver resection, recurrence of disease has been reported in up to two-thirds of patients, with half of these occurring in the remnant liver [30, 31]. This has lead to numerous studies highlighting the benefit of pre- and post-operative chemotherapy in improving outcomes after hepatectomy for metachronous CRLM. Non-randomized trials of adjuvant treatment with 5-fluorouracil (5-FU) based chemotherapy or the use of hepatic arterial infusion of floxuridine (FUDR) have demonstrated improvement in survival and decreased rates of recurrence [32-34] However, the recently concluded European Organisation for Research and Treatment of Cancer (EORTC) intergroup trial 40,983 failed to demonstrate any benefit for perioperative chemotherapy with liver 
Table 2 Characteristics of studies of simultaneous versus staged hepatectomy in sCRLM

\begin{tabular}{|c|c|c|c|c|c|c|c|c|c|c|c|}
\hline \multirow[t]{2}{*}{ Author } & \multirow[t]{2}{*}{ Year } & \multicolumn{4}{|c|}{ Simultaneous resection } & \multicolumn{4}{|c|}{ Staged resection } & \multicolumn{2}{|c|}{ Neoadjuvant chemotherapy } \\
\hline & & Patients & $\begin{array}{l}\text { Mean } \\
\text { age }\end{array}$ & $\begin{array}{l}\text { Liver } \\
\text { mets }\end{array}$ & $\begin{array}{l}\text { Major liver } \\
\text { resection (\%) }\end{array}$ & Patients & $\begin{array}{l}\text { Mean } \\
\text { age }\end{array}$ & $\begin{array}{l}\text { Liver } \\
\text { mets }\end{array}$ & $\begin{array}{l}\text { Major liver } \\
\text { resection }(\%)\end{array}$ & One stage & Staged \\
\hline Vogt [53] & 1991 & 19 & 56.5 & 1.79 & 32 & 17 & 56.5 & 1.24 & 53 & NA & NA \\
\hline Jaeck [54] & 1999 & 28 & 56 & - & 32 & 31 & 60 & - & 52 & NA & NA \\
\hline Weber [55] & 2003 & 35 & 58.0 & 1.9 & 31 & 62 & 60.0 & 3.7 & 52 & $44 \%$ & $61.2 \%$ \\
\hline Chua [56] & 2004 & 64 & 63.0 & - & 16 & 32 & 61.0 & - & 40 & NA & NA \\
\hline Tanaka [57] & 2004 & 39 & 64.0 & 2.2 & 13 & 37 & 65.0 & 5.3 & 59 & NA & NA \\
\hline Minagawa [58] & 2006 & 142 & - & - & 11 & 18 & - & - & 38 & NA & NA \\
\hline Capossotti $[17 \bullet]$ & 2007 & 70 & 64.9 & - & 34 & 57 & 60.8 & - & 56 & NA & NA \\
\hline Thelen [22] & 2007 & 57 & 60.0 & - & 38 & 179 & 59.7 & - & 79 & $7.5 \%$ & NA \\
\hline Reddy [59] & 2007 & 135 & 57.0 & 1 & 26 & 475 & 58.0 & 2 & 61 & $60.7 \%$ & $79.2 \%$ \\
\hline Yan [60] & 2007 & 73 & 60.0 & 4 & 74 & 30 & 59.0 & 3 & 73 & NA & NA \\
\hline Slupski [61] & 2008 & 28 & 59.4 & 2.9 & 29 & 61 & 60.2 & 3.8 & 48 & NA & NA \\
\hline Martin [16] & 2009 & 70 & 58 & 3 & 47 & 160 & 61 & 3 & 40 & $52 \%$ & $70 \%$ \\
\hline Brouquet [62] & 2010 & 43 & 58 & 2 & 35 & 72 & 56 & 3 & 67 & $11 \%$ & $59 \%$ \\
\hline De Haas [63] & 2010 & 55 & 56.0 & 2 & - & 173 & 58.0 & 3 & - & $24 \%$ & $95.4 \%$ \\
\hline Luo [64] & 2010 & 129 & 58.0 & - & 32 & 276 & 60.0 & - & 38 & $40 \%$ & $61.2 \%$ \\
\hline Moug [65] & 2010 & 32 & 69 & - & 22 & 32 & 67 & - & 22 & $40.6 \%$ & $53.1 \%$ \\
\hline Petri [66] & 2010 & 14 & - & - & 0 & 29 & - & - & 21 & NA & NA \\
\hline van der Pool [67] & 2010 & 8 & - & 1 & - & 29 & - & 2 & - & NA & NA \\
\hline Slesser $\left[20^{\bullet}\right]$ & 2013 & 36 & 55.5 & - & 64 & 76 & 62.0 & - & 79 & $88 \%$ & $99 \%$ \\
\hline Mayo [23••] & 2013 & 329 & 60.0 & - & 23.7 & 675 & 61.0 & - & 38.6 & $21.6 \%$ & $20.1 \%$ \\
\hline
\end{tabular}

$N A$ not available

resection of CRLM [35••]. This trial was a randomized, controlled, parallel-group, phase 3 study involving 78 hospitals in which patients with CRLMs (up to 4) were randomly assigned to surgery alone or perioperative FOLFOX4, (5-FU, folinic acid and oxaliplatin) chemotherapy. The perioperative chemotherapy regimen consisted of six 14-day cycles before and after hepatectomy. Despite initial reports that perioperative chemotherapy had improved progression-free survival, the long-term results indicate that there is no difference in 5-year OS. Another recent study by Faron et al. [36] examined the impact of preand post-operative chemotherapy separately in patients with resectable CRLM. This study found that while pre-operative chemotherapy did not improve OS, postoperative chemotherapy was an independent predictor of increased OS and DFS. The current data do not indicate that pre-operative chemotherapy has a proven role prior to hepatectomy for CRLM, although this question merits further study, especially with regards to the specific benefit of adjuvant therapy.

The effect of chemotherapy-related hepatic toxicity must also be considered when pre-operative chemotherapy is used. Regimens containing irinotecan have been associated with steatohepatitis in up to $66 \%$ of patients, and hepatic sinusoidal abnormalities have been seen with oxaliplatin based regimens $[37,38]$. The addition of bevacizumab has been demonstrated to increase the frequency of tumor regression when utilized in conjunction with oxaliplatin or irinotecan, but is associated with increased toxicity and the need to delay hepatectomy for up to 4 weeks after the last dose due to its prolonged half life $[39,40]$.

In patients with sCRLM that are deemed resectable, the National Comprehensive Cancer Network guidelines recommend either simultaneous resection followed by adjuvant therapy-with FOLFOX or CapeOx (capecitabine, oxaliplatin) —or neoadjuvant therapy for 2-3 months prior to operation [41]. Recommended neoadjuvant regimens include FOLFIRI (folinic acid, 5-FU and irinotecan), FOLFOX, or CapeOx with or without bevacizumab. If the tumor is KRAS wild-type, the use of FOLFOX or FOLFIRI with or without panitumumab or cetuximab (monoclonal antibodies against EGFR) may be considered [41, 42].

\section{Adjunctive Strategies for Initially Unresectable Disease}

Seventy-five to $85 \%$ of patients with sCRLM have unresectable disease due to either the size, location, multifocality of metastases or have an inadequate projected FLR [43]. Several established and emerging therapeutic strategies 
have been used to attempt to convert patients to resectability. In patients deemed to have unresectable disease, the use of systemic chemotherapy has demonstrated conversion of $15-20 \%$ of patients to undergo surgical resection with a 5 -year OS of 33-42\% $[44,45]$. In this clinical scenario, the regimens outlined in the previous section are recommended by the NCCN. In addition, there are many novel therapeutic combinations that have been described, but are beyond the scope of this review.

The ability to safely perform a major hepatectomy has expanded the extent of liver resection that surgeons may consider in order to extirpate all metastatic lesions. A fundamental factor governing this decision, however, is the FLR. In general, a goal of retaining 20-25\% of a normal liver, or 30-35\% of liver in patients treated with chemotherapy is necessary for the FLR to be deemed adequate. This can be assessed with volumetric CT imaging. The most common maneuvers employed to enhance the FLR are either radiologic portal vein embolization or operative portal vein ligation of the side of the liver that is planned for resection. This approach allows for hypertrophy of the residual liver in four to 6 weeks for adequate FLR when an extended hepatectomy may be necessary to achieve an R0 resection.

Another approach to managing bi-lobar disease is a twostage hepatectomy, as this allows for residual liver regeneration in the 6-8 weeks between hepatectomies. Extreme surgical approaches involving ex vivo hepatic resection, vascular exclusion, and hypothermic perfusion have also been reported, but these are performed only in highly specialized circumstances in close collaboration with liver transplantation teams.

Associating liver partition and portal vein ligation for staged hepatectomy (ALPPS) is a technique that has gained much attention in recent years and combines aspects of the techniques outlined above. This technique is a modification of both two-stage hepatectomy and extended right hepatectomy in which the right portal vein is ligated and the hepatic parenchyma is split in situ during the initial operation [47•]. The extended right lobe is covered with a plastic bag to prevent formation of adhesions in the interval between operations. Regeneration of hepatic parenchyma is measured at post-operatively via volumetric CT (at a median of 9 days in the original study), and if the FLR is adequate then the second operation is scheduled for the following day. During the second operation the extended right hepatic lobe is removed by ligating the right hepatic artery, right and middle hepatic veins and bile ducts The initial results of this approach were reported by Schnitzbauer et al. [47•] and indicated that the left lateral section volume increased $74 \%$ at a median of 9 days after the initial operation. A subsequent study by de Santibanes et al. [48] demonstrated 40-80\% hypertrophy of the FLR 6 days after the initial operation [48]. Several groups have adopted and refined this technique, and it remains an area of expanded use and surgical innovation.

Ablative therapies offer another strategy to downsize or eliminate metastatic lesions. Ablative approaches may be used in circumstances when the patient may not tolerate a liver resection, or in conjunction with surgical resection in the presence of bi-lobar disease when attempting to achieve a curative resection, particularly when extended hepatectomy may not allow for an adequate FLR.

Cryotherapy induces tumor necrosis through the circulation of liquid nitrogen through a metal probe that is inserted either percutaneously or intra-operatively (laparoscopic or open) and the resultant rapid freezing of tissue leads to tumor destruction. Its use has diminished in recent years, however, due to higher complication rates and technical limitations compared with radiofrequency ablation (RFA) [49].

The most widely used thermoablative modality is RFA. In this technique, an electrode is inserted into the tumor, and then a high frequency alternating electrical current generates frictional heat resulting in coagulation necrosis of tumor tissue. A single electrode can ablate a two to three $\mathrm{cm}$ lesion with a $1-\mathrm{cm}$ margin, but larger tumors require either multiple electrode placements or the deployment of an electrode array in order to encompass the entire area [50]. The utility of RFA in patients with unresectable disease has not been well established; however, an American Society of Clinical Oncology (ASCO) clinical evidence review of the existing data on RFA concluded that there is currently a compelling need for more study in this area [51].

Microwave ablation is another modality that can be employed intraoperatively or through image guidance. Tissue heating results from the agitation of water molecules, resulting in hyperthermia and coagulation necrosis. In a trial of microwave ablation versus hepatic resection for CRLMs performed by Shibata et al. [52] demonstrated a similar OS between the groups.

Select patients that present with pulmonary metastases in addition to CRLM may be considered for combined or staged pulmonary and liver metastasectomy. These patients are evaluated in conjunction with a thoracic surgeon, usually after receiving a period of systemic chemotherapy verifying the lack of progression of disease, if all the pulmonary disease can be removed without compromising pulmonary function [46].

\section{Conclusion}

Management of patients who present with sCRLM is complex and evolving at a rapid pace. There is no clear 
evidence to indicate that there is an optimal approach to timing of resection of the primary tumor or metastatic disease, and the evidence that is available indicates that there is no significant difference in outcomes between approaches. The availability of effective chemotherapy regimens and innovative surgical and ablative approaches allows for an array of therapeutic modalities to optimize patients outcomes. The decision on operative timing and approach, then, remains largely dependent on the patient's response to systemic therapy, the surgeon's determination of disease resectability and patient symptomatology and fitness.

\section{Compliance with Ethics Guidelines}

Conflict of Interest Jason A. Castellanos and Nipun B. Merchant declare that they have no conflict of interest.

Human and Animal Rights and Informed Consent This article does not contain any studies with human or animal subjects performed by any of the authors.

\section{References}

Recently published papers of particular interest have been highlighted as:

- Of importance

•- Of major importance

1. Siegel R, Ma J, Jemal A. Cancer statistics, 2014. CA Cancer J Clin. 2014;62:9-29.

2. Jessup JM, McGinnis LS, Steele GD Jr, Menck HR, Winchester DP. The national cancer data base: report on colon cancer. Cancer. 1996;78:918-26.

3. - Adams RB, Aloia TA, Loyer E, et al. Selection for hepatic resection of colorectal liver metastases: expert consensus statement. HPB (Oxford) 2013;15:91-103. Guidelines for hepatic resection for patients with CRC liver metastases as outlined by the Consensus Conference on Multidisciplinary Treatment of Colorectal Cancer Liver Metastases in January, 2012.

4. - Adam R, de Haas RJ, Wicherts DA, et al. Is hepatic resection justified after chemotherapy in patients with colorectal liver metastases and lymph node involvement? J Clin Oncol 2008;26:3672-80. This study demonstrated that celiac and/or para-aortic regional lymph node metastases indicate a cohort of patients with poor prognosis.

5. - Jaeck D, Nakano H, Bachellier P, et al. Significance of hepatic pedicle lymph node involvement in patients with colorectal liver metastases: a prospective study. Ann Surg Oncol 2002;9:430-8. A study of 160 patients undergoing hepatectomy for CRLM that demonstrated patients with portal lymph node metastases should be considered for resection.

6. Gallagher DJ, Kemeny N. Metastatic colorectal cancer: from improved survival to potential cure. Oncology. 2010;78:237-48.

7. • Pawlik TM, Scoggins CR, Zorzi D, et al. Effect of surgical margin status on survival and site of recurrence after hepatic resection for colorectal metastases. Ann Surg 2005;241:715-22, discussion 22-4. A multicenter retrospective study of 557 patients that underwent hepatectomy for CRLM which demonstrated that $R O$ resection is associated with significantly improved OS and decreased recurrence.

8. - Scheele J, Stang R, Altendorf-Hofmann A, Paul M. Resection of colorectal liver metastases. World J Surg 1995;19:59-71. An early retrospective review of 1209 patients with CRLM which demonstrated survival benefit for hepatectomy.

9. - Stangl R, Altendorf-Hofmann A, Charnley RM, Scheele J. Factors influencing the natural history of colorectal liver metastases. Lancet 1994;343:1405-10. This study provided an early framework to determine benefit of hepatectomy for CRLM.

10. Bismuth H, Castaing D, Traynor O. Surgery for synchronous hepatic metastases of colorectal cancer. Scand J Gastroenterol Suppl. 1988;149:144-9.

11. - Bolton JS, Fuhrman GM. Survival after resection of multiple bilobar hepatic metastases from colorectal carcinoma. Ann Surg 2000;231:743-51. A retrospective analysis of 165 patients which concludes that hepatectomy should be delayed if CRLMs are compex due to higher postoperative mortality.

12. • Lambert LA, Colacchio TA, Barth RJ. Interval hepatic resection of colorectal metastases improves patient selection. Curr Surg 2000;57:504. This retrospective review evaluated results of reevaluation for resectability of CRLM after colectomy.

13. - Scheele J. Hepatectomy for liver metastases. Br J Surg 1993;80:274-6. A review of hepatectomy for CRLM which outlines an argument for delayed hepatectomy.

14. Alberts SR, Poston GJ. Treatment advances in liver-limited metastatic colorectal cancer. Clin Colorectal Cancer. 2011;10: 258-65.

15. Mentha G, Majno P, Terraz S, et al. Treatment strategies for the management of advanced colorectal liver metastases detected synchronously with the primary tumour. Eur J Surg Oncol. 2007;33(Suppl 2):S76-83.

16. •• Martin RC, 2nd, Augenstein V, Reuter NP, Scoggins CR, McMasters KM. Simultaneous versus staged resection for synchronous colorectal cancer liver metastases. J Am Coll Surgeons 2009;208:842-50; discussion 50-2. A retrospective study of outcomes of patients with sCRLM which demonstrated decreased hospital stay with no difference in morbidity and mortality with synchronous resection.

17. - Capussotti L, Ferrero A, Vigano L, Ribero D, Lo Tesoriere R, Polastri R. Major liver resections synchronous with colorectal surgery. Ann Surg Oncol 2007;14:195-201. An evaluation of short-term outcomes of simultaneous major hepatectomy and colorectal surgery for sCRLM.

18. de Santibanes E, Lassalle FB, McCormack L, et al. Simultaneous colorectal and hepatic resections for colorectal cancer: postoperative and longterm outcomes. J Am Coll Surg. 2002;195: 196-202.

19. - Martin R, Paty P, Fong Y, et al. Simultaneous liver and colorectal resections are safe for synchronous colorectal liver metastasis. J Am Coll Surgeons 2003;197:233-41; discussion 41-2. A single institution comparison of delayed vs. simultaneous resection of $S C R L M$.

20. - Slesser AA, Chand M, Goldin R, Brown G, Tekkis PP, Mudan S. Outcomes of simultaneous resections for patients with synchronous colorectal liver metastases. Eur J Surg Oncol 2013;39:1384-93. A single institution study of 112 patients that demonstrated similar short-term and long-term outcomes with simultaneous and delayed surgical approaches to SCRLM.

21. - Nordlinger B, Guiguet M, Vaillant JC, et al. Surgical resection of colorectal carcinoma metastases to the liver. A prognostic scoring system to improve case selection, based on 1568 patients. Association Francaise de Chirurgie. Cancer 1996;77:1254-62. Data from 1568 patients with resected CRLM that stratified two year survival rates by risk factors (age, size of largest CRLM, 
CEA level, stage or primary, DFS, number of liver nodules, resection margin).

22. Thelen A, Jonas S, Benckert C, et al. Simultaneous versus staged liver resection of synchronous liver metastases from colorectal cancer. Int J Colorectal Dis. 2007;22:1269-76.

23. •• Mayo SC, Pulitano C, Marques H, et al. Surgical management of patients with synchronous colorectal liver metastasis: a multicenter international analysis. J Am Coll Surgeons 2013;216:707-16; discussion 16-8. A multi-institution review of outcomes after either staged or simultaneous resection of SCRLM.

24. •• Slesser AA, Simillis C, Goldin R, Brown G, Mudan S, Tekkis PP. A meta-analysis comparing simultaneous versus delayed resections in patients with synchronous colorectal liver metastases. Surgical oncology 2013;22:36-47. A meta-analysis of 24 studies encompassing 3,159 patients that underwent resection for sCRLM.

25. • Yin Z, Liu C, Chen Y, et al. Timing of hepatectomy in resectable synchronous colorectal liver metastases (SCRLM): Simultaneous or delayed? Hepatology 2013;57:2346-57. A metaanalysis of studies encompassing 2,880 patients that underwent resection for $S C R L M$.

26. - Mentha G, Roth AD, Terraz S, et al. 'Liver first' approach in the treatment of colorectal cancer with synchronous liver metastases. Dig Surg 2008;25:430-5. A retrospective review of 35 patients that underwent staged resection with hepatectomy first.

27. Van Dessel E, Fierens K, Pattyn P, et al. Defining the optimal therapy sequence in synchronous resectable liver metastases from colorectal cancer: a decision analysis approach. Acta Chir Belg. 2009;109:317-20.

28. Mentha G, Majno PE, Andres A, Rubbia-Brandt L, Morel P, Roth AD. Neoadjuvant chemotherapy and resection of advanced synchronous liver metastases before treatment of the colorectal primary. Br J Surg. 2006;93:872-8.

29. de Jong MC, van Dam RM, Maas M, et al. The liver-first approach for synchronous colorectal liver metastasis: a 5-year single-centre experience. HPB (Oxford). 2011;13:745-52.

30. de Jong MC, Pulitano C, Ribero D, et al. Rates and patterns of recurrence following curative intent surgery for colorectal liver metastasis: an international multi-institutional analysis of 1669 patients. Ann Surg. 2009;250:440-8.

31. Fong Y, Salo J. Surgical therapy of hepatic colorectal metastasis. Semin Oncol. 1999;26:514-23.

32. Kemeny MM, Adak S, Gray B, et al. Combined-modality treatment for resectable metastatic colorectal carcinoma to the liver: surgical resection of hepatic metastases in combination with continuous infusion of chemotherapy-an intergroup study. J Clin Oncol. 2002;20:1499-505.

33. Lorenz M, Muller HH, Schramm H, et al. Randomized trial of surgery versus surgery followed by adjuvant hepatic arterial infusion with 5-fluorouracil and folinic acid for liver metastases of colorectal cancer. German Cooperative on Liver Metastases (Arbeitsgruppe Lebermetastasen). Ann Surg. 1998;228:756-62.

34. Kemeny N, Huang Y, Cohen AM, et al. Hepatic arterial infusion of chemotherapy after resection of hepatic metastases from colorectal cancer. N Engl J Med. 1999;341:2039-48.

35. - Nordlinger B, Sorbye H, Glimelius B, et al. Perioperative FOLFOX4 chemotherapy and surgery versus surgery alone for resectable liver metastases from colorectal cancer (EORTC 40983): long-term results of a randomised, controlled, phase 3 trial. Lancet Oncol 2013;14:1208-15. Final results of EORTC 40983 indicated no difference in OS with the addition of perioperative FOLFOX4 vs. surgery alone in patients with resectable CRLM.

36. Faron M, Chirica M, Tranchard H, et al. Impact of preoperative and postoperative FOLFOX chemotherapies in patients with resectable colorectal liver metastasis. J Gastrointest Canc. 2014. doi:10.1007/s12029-014-9594-y.

37. Rubbia-Brandt L, Audard V, Sartoretti P, et al. Severe hepatic sinusoidal obstruction associated with oxaliplatin-based chemotherapy in patients with metastatic colorectal cancer. Ann Oncol. 2004;15:460-6.

38. Kooby DA, Fong Y, Suriawinata A, et al. Impact of steatosis on perioperative outcome following hepatic resection. J Gastrointest Surg. 2003;7:1034-44.

39. Reddy SK, Morse MA, Hurwitz HI, et al. Addition of bevacizumab to irinotecan- and oxaliplatin-based preoperative chemotherapy regimens does not increase morbidity after resection of colorectal liver metastases. J Am Coll Surg. 2008;206:96-106.

40. Walter $\mathrm{H}$, Thomas AL. Liver resection following FOLFOXIRI plus bevacizumab: a detailed pathological review. Br J Cancer. 2013;108:2417-8

41. NCCN Clinical Practice Guidelines in Oncology: Colon Cancer. Version 3, 2014. Accessed 21 March 2014, at NCCN.org.

42. NCCN Clinical Practice Guidelines in Oncology: Rectal Cancer. Version 3, 2014. Accessed 21 March 2014, at NCCN.org.

43. Adam R, Miller R, Pitombo M, et al. Two-stage hepatectomy approach for initially unresectable colorectal hepatic metastases. Surg Oncol Clin N Am 2007;16:525-36, viii.

44. Adam R, Delvart V, Pascal G, et al. Rescue surgery for unresectable colorectal liver metastases downstaged by chemotherapy: a model to predict long-term survival. Ann Surg 2004;240:644-57; discussion 57-8.

45. Wicherts DA, Miller R, de Haas RJ, et al. Long-term results of two-stage hepatectomy for irresectable colorectal cancer liver metastases. Ann Surg. 2008;248:994-1005.

46. Gallinger S, Biagi JJ, Fletcher GG, Nhan C, Ruo L, McLeod RS. Liver resection for colorectal cancer metastases. Curr Oncol. 2013;20:e255-65.

47. - Schnitzbauer AA, Lang SA, Goessmann H, et al. Right portal vein ligation combined with in situ splitting induces rapid left lateral liver lobe hypertrophy enabling 2-staged extended right hepatic resection in small-for-size settings. Ann Surg 2012;255:405-14. The initial report of the ALPPS technique.

48. de Santibanes E, Alvarez FA, Ardiles V. How to avoid postoperative liver failure: a novel method. World J Surg. 2012;36:125-8.

49. Garrean S, Hering J, Helton WS, Espat NJ. A primer on transarterial, chemical, and thermal ablative therapies for hepatic tumors. Am J Surg. 2007;194:79-88.

50. Curley SA. Radiofrequency ablation of malignant liver tumors. Ann Surg Oncol. 2003;10:338-47.

51. Wong SL, Mangu PB, Choti MA, et al. American Society of Clinical Oncology 2009 clinical evidence review on radiofrequency ablation of hepatic metastases from colorectal cancer. J Clin Oncol. 2010;28:493-508.

52. Shibata T, Niinobu T, Ogata N, Takami M. Microwave coagulation therapy for multiple hepatic metastases from colorectal carcinoma. Cancer. 2000;89:276-84.

53. Vogt P, Raab R, Ringe B, Pichlmayr R. Resection of synchronous liver metastases from colorectal cancer. World J Surg. 1991;15:62-7.

54. Jaeck D, Bachellier P, Weber JC, et al. Surgical strategy in the treatment of synchronous hepatic metastases of colorectal cancers. Analysis of a series of 59 operated on patients. Chirurgie; memoires de l'Academie de chirurgie. 1999;124:258-63.

55. Weber JC, Bachellier P, Oussoultzoglou E, Jaeck D. Simultaneous resection of colorectal primary tumour and synchronous liver metastases. Br J Surg. 2003;90:956-62.

56. Chua HK, Sondenaa K, Tsiotos GG, Larson DR, Wolff BG, Nagorney DM. Concurrent vs. staged colectomy and hepatectomy for primary colorectal cancer with synchronous hepatic metastases. Dis Colon Rectum. 2004;47:1310-6. 
57. Tanaka K, Shimada H, Matsuo K, et al. Outcome after simultaneous colorectal and hepatic resection for colorectal cancer with synchronous metastases. Surgery. 2004;136:650-9.

58. Minagawa M, Yamamoto J, Miwa S, et al. Selection criteria for simultaneous resection in patients with synchronous liver metastasis. Arch Surg 2006;141:1006-12; discussion 13.

59. Reddy SK, Pawlik TM, Zorzi D, et al. Simultaneous resections of colorectal cancer and synchronous liver metastases: a multiinstitutional analysis. Ann Surg Oncol. 2007;14:3481-91.

60. Yan TD, Chu F, Black D, King DW, Morris DL. Synchronous resection of colorectal primary cancer and liver metastases. World J Surg. 2007;31:1496-501.

61. Slupski M, Wlodarczyk Z, Jasinski M, Masztalerz M, Tujakowski J. Outcomes of simultaneous and delayed resections of synchronous colorectal liver metastases. Can J Surg. 2009;52: E241-4.

62. Brouquet A, Mortenson MM, Vauthey JN, et al. Surgical strategies for synchronous colorectal liver metastases in 156 consecutive patients: classic, combined or reverse strategy? J Am Coll Surg. 2010;210:934-41.
63. de Haas RJ, Adam R, Wicherts DA, et al. Comparison of simultaneous or delayed liver surgery for limited synchronous colorectal metastases. Br J Surg. 2010;97:1279-89.

64. Luo Y, Wang L, Chen C, et al. Simultaneous liver and colorectal resections are safe for synchronous colorectal liver metastases. J Gastrointest Surg. 2010;14:1974-80.

65. Moug SJ, Smith D, Leen E, Roxburgh C, Horgan PG. Evidence for a synchronous operative approach in the treatment of colorectal cancer with hepatic metastases: a case matched study. Eur J Surg Oncol. 2010;36:365-70.

66. Petri A, Hohn J, Balogh A, Kovach K, Andrasi L, Lazar G. Surgical treatment of liver metastasis in colorectal cancer with simultaneous liver resection. Magyar Onkologia. 2010;54:125-8.

67. van der Pool AE, de Wilt JH, Lalmahomed ZS, Eggermont AM, Ijzermans JN, Verhoef C. Optimizing the outcome of surgery in patients with rectal cancer and synchronous liver metastases. Br J Surg. 2010;97:383-90. 